\title{
Escala para la Evaluación de la Educación frotística (EEEA)
}

\author{
Scale for Evaluation of Artistic Education
}

\author{
Irasema del Pilar Castell Ruiz \\ Martha Frías Armenta \\ Antonio Medina Rivilla
}

\section{Resumen}

El objetivo del presente trabajo es presentar el proceso de diseño y validación de la Escala para la Evaluación de la Educación Artística (EEEA). Se elaboró un cuestionario ex profeso con datos sociodemográficos y con preguntas específicas sobre la formación en educación artística; éste se dividió en dos secciones: I) Sección institución y II) Sección alumno(a). La primera sección fue conformada por las sub escalas: 1) características de la institución, 2) características del aula, 3) actividades artísticas, y 4) estructura del programa. Las cuatro se adaptaron de la Escala de Evaluación de Contextos Educativos Infantiles (ECERS-R, Harms \& Clifford, 1980). Para la sección alumno(a) se construyeron cuatro sub escalas: 1) características del alumno con sus respectivas dimensiones (académicas, creatividad, artísticas, inteligencias múltiples, liderazgo, comportamientos éticos, inteligencia emocional y salud), 2) características de los padres, 3) características del maestro(a), y 4) interacción, con un total de 135 ítems. La escala EEEA se aplicó a 50 maestros y 50 padres de niños sordos que cursaban educación básica. Los valores de las alfas de las escalas analizadas revelaron consistencia interna dado que todas fueron mayores a.75.

\section{Abstract}

This study was aimed at designing and validating the Artistic Education Evaluation Scale (EEEA). The survey investigated demographic aspects, and also included ítems about courses and artistic education background. It was divided into two sections: institution and students. The former investigated 1) characteristics of the institution, 2) characteristics of the classroom, 3) artistic activities, and 4) program structure. The four sections were adapted from the Early Childhood Environment Rating Scale Revised (ECERS-R, Harms \& Clifford, 1980). The students section comprised four sub-scales: 1) students' (academic, creativity, artistic, multiple intelligences, ethics, and health dimensions) characteristics, 2) parents' characteristics, 3) teachers' characteristics, and 4) interactions. The sample included 50 teachers and 50 parents of deaf children attending elementary schools. Cronbach's alpha of all sub-scales produced values higher than .75, indicating internal consistency.

Palabras Clave: Evaluación, educación artística, escala, confiabilidad, formación.

Keywords: Evaluation, artistic education, scale, reliability, formation. 
La educación tiene como objetivo primordial el avance permanente de la persona y de la sociedad en general, se pudiera considerar un instrumento para el progreso de la humanidad y para enfrentar los numerosos desafíos del desarrollo así como los ideales de paz, libertad y justicia social (Delors, 2009). Por su parte, los gobiernos intentan dar cobertura universal a tal objetivo, buscando que la mayor parte de la población se integre a la vida escolarizada (Plan Nacional de Desarrollo, 2006). Internacionalmente, los sistemas educativos han ido extendiendo de manera gradual el carácter obligatorio de la formación básica. En la actualidad, ésta comprende en algunos países no sólo la primaria y la secundaria, sino también se ha incorporado el preescolar. En el caso de México, las modificaciones se han realizado con el fin de sentar las bases y lograr un mejor aprovechamiento en el alumnado. Este beneficio incluye mejorar al ser humano para que contribuya activamente en la sociedad.

No obstante, la Alianza por la Calidad de la Educación (ACE, 2010) considera que una formación conlleva reformas y transformaciones, entre otras cosas, de los planes, los programas y las materias de estudio. Una de las reformas a los planes educativos es la incorporación de la instrucción artística como una asignatura fundamental en el currículo de la enseñanza básica. Sin embargo, aún se considera erróneamente que las actividades artísticas son para pasar el tiempo o para entretener. Para Gardner (2010), el éxito de la educación depende de la participación de la comunidad, el currículo, el desarrollo profesional, la educación de profesores, además de la evaluación. En atención a estos dos últimos aspectos, en nuestro país la cultura de la evaluación es casi inexistente (Frías \& Corral, 2009).

Por otra parte, tampoco se cuenta con instrumentos para evaluar la calidad y efectos de la educación artística; no se realizan investigaciones ni actividades que demuestren la contribución del arte en la educación. El arte pudiera fomentar el desarrollo estético, socio emocional, sociocultural, cognoscitivo y el progreso escolar. Adicionalmente, no se contempla la asignatura de Educación artística en el currículo de la carrera magisterial, mientras que la capacitación en esta materia para maestros(as) normalistas deja mucho que desear. Al respecto, el Instituto Federal de Acceso a la Información (IFAI, 20082009), publicó que los maestros con certificados de nivel primaria, en un mayor porcentaje de los estados de México, Guanajuato, Sonora y Veracruz, son los que enseñan a los niños a leer, escribir, sumar y restar. Adicionalmente, son responsables de ejercer los recursos públicos en sus escuelas, y de las actividades computacionales, deportivas y artísticas. Por ende, el panorama resulta poco prometedor para el proceso de enseñanza-aprendizaje en México.

De acuerdo a Destrempes-Márquez y Lafleur (2003) aprender requiere de tres precurrentes básicas: prestar atención, imitación y seguimiento de instrucciones; además está compuesto de varias etapas. Primero, la información se registra en el cerebro (etapa de recepción o percepción), posteriormente, ésta debe ser decodificada y comprendida (etapa de la integración). Una vez que la información ha sido registrada e integrada, debe ser memorizada para poder usarla otra vez y, finalmente, ser comunicada a través del cerebro hacia el exterior o traducirse en una acción del individuo (producción o ejecución).

El arte puede refinar la percepción sensorial, animar la exploración y el paso por dichas etapas, enriquecer las experiencias de aprendizaje y mejorar el rendimiento académico. Al respecto, Passenger, Stuart, y Terrel (2003) en su investigación de identificaron de redes neuronales (sistemas de conexión entre las áreas del cerebro) encontró que el entrenamiento en artes influencia positivamente la atención. De ahí, la importancia de contemplar en el currículo el arte dentro de los contenidos de la educación; no sólo en la básica sino hasta la superior. 
"La Alianza por la Calidad de la Educación (2008), establece la necesidad de impulsar una reforma sobre los enfoques, asignaturas y contenidos de la educación básica, con el propósito de formar ciudadanos íntegros capaces de desarrollar todo su potencial" (Programa Sectorial de Educación, PROSEDU, 2007-2012, p.22). El arte puede ayudar a ese desarrollo integral, ya que tiene un gran impacto en diversas áreas de la vida e inteligencia pueril. Sin embargo, no se han realizado en México, concretamente en el estado de Sonora, evaluaciones científicas para detectar los efectos que la educación artística brinda en las múltiples inteligencias tanto formales como informales. Si se hicieran tales evaluaciones en nuestro país y en nuestro estado, éstas se podrían tomar como base para seleccionar acciones que puedan mejorar la educación de niños y adolescentes. Gento (2002), propone recrear los entornos educativos con tintes más humanos, relajados, optimistas y con sentido del humor. El planteamiento de dicho autor, podría dar oportunidad de crear repertorios académicos en los cuales se desarrolle creatividad, inteligencias múltiples, capacidad de liderazgo, inteligencia emocional, comportamientos éticos e incluso la salud de los educandos.

La educación artística impacta en el desarrollo de múltiples inteligencias; cuestión que ha sido estudiada en otros países (Goleman, 2005; Greenberg, 2003). A Goleman (2005), le preocupó la alfabetización emocional de los niños, cómo implementarla en el ámbito escolar y el impacto que pudiera tener en ellos. Greenberg (2003), por su parte, confeccionó el programa Promoviendo Estrategias de Pensamiento Alternativo (PHATS ${ }^{1}$ ), que pretendía inculcar en los niños sordos la utilización del lenguaje para cobrar conciencia, examinar y gestionar más apropiadamente sus emociones y las emociones de los demás. Para Gardner (2006), la cultura americana se ha concentrado durante mucho tiempo en las habilidades académicas e ignora la inteligencia emocional, que también tiene una enorme importancia para el destino personal de los niños. Un Coeficiente Intelectual $(\mathrm{Cl})$ elevado no es garantía de bienestar, prestigio, ni felicidad en la vida. La argumentación de este autor no es la excepción para el sistema educativo mexicano.

No obstante, el énfasis en las habilidades académicas y los resultados de la evaluación de tres competencias: lectura, matemáticas y ciencias, realizado en el marco del Programa Internacional para la Evaluación de los Alumnos (PISA ${ }^{2}$ y de la Organización para la Cooperación y el Desarrollo Económicos (OCDE), mostró que el $50 \%$ de los alumnos mexicanos de 15 años se ubicó en los niveles cero y uno, los más bajos del rendimiento escolar en estas habilidades, es decir, los jóvenes mexicanos están poco calificados para acceder a estudios superiores o, por lo menos, para resolver problemas elementales (PISA 2006).

Para calificar los atributos de programas, políticas públicas y determinar la calidad educativa se requiere una valoración que implica el análisis sistemático de los efectos de una política pública o programa social, a partir de criterios determinados y en correspondencia con los objetivos propuestos y esperados en los grupos sociales favorecidos (Jacinto \& Gallart, 1998). Para Rossi y Freeman (1985), la evaluación es "la utilización del método científico para valorar la conceptualización, el diseño, la implementación e impacto de los programas de intervención." Por su parte, Frías y Corral (2001), definen la evaluación como "la estimación, a través del método científico, de la congruencia de los programas sociales de intervención".

Los resultados vertidos en el informe PISA 2006, indican que la evaluación tiene un potencial de alto impacto para la enseñanza y para la política pública porque: a) provee a los

\footnotetext{
${ }^{1}$ Por sus siglas en inglés.

2 Por sus siglas en inglés.
} 
maestros y a los creadores de políticas públicas de una perspectiva sobre cómo mejorar la calidad, equidad y eficiencia de la educación; y b) amplía el rango de competencias a través del cual se evalúa la calidad. Por su parte, la calidad de la educación se enfoca en el avance, en tanto, la evaluación establece las herramientas metodológicas para el análisis de aquélla y el establecimiento de las propuestas para mejorarla (Gento, 2008). El problema es que resulta imprescindible medir el impacto de las políticas públicas relacionadas con la educación y el arte; así como la calidad de las instituciones y las aulas donde se implementa la asignatura de Educación artística, los programas, además, de las actividades artísticas que se realizan en las instituciones educativas, la concepción y la finalidad que se persigue al enseñar arte en cualquiera de sus modalidades.

En la actualidad, existe un buen número de instrumentos para evaluar varios rubros correspondientes a la educación. Sin embargo, en la literatura no se observan herramientas que midan la efectividad de la Educación artística ni el impacto que ésta pudiera tener en el niño(a). Aunado a esto, la comprensión de los efectos de la instrucción artística que los niños(as) reciben por parte de la institución educativa y de sus maestros es muy limitada, debido, en parte, a las dificultades asociadas a la adaptación o desarrollo de medidas objetivas. De hecho, muchos instrumentos enfocados en la educación, ampliamente utilizados para realizar evaluaciones, no poseen un adecuado ajuste a las variaciones culturales.

Tal situación es de considerar dado que se requiere tener la mayor certeza posible de que se mide lo que se quiere medir y que, además, se mide con precisión. Es decir, se cuente con instrumentos válidos y confiables, con el fin de que las conclusiones obtenidas en las investigaciones y en las evaluaciones realizadas en diversos ámbitos, entre ellos el educativo, se caractericen por ser válidas y consistentes con otros instrumentos, otras poblaciones y otras culturas. Para ello es importante considerar, du- rante el diseño de un instrumento para medir el impacto de la formación artística en los ámbitos educativos, la selección de aquellos indicadores que reflejen fielmente un determinado constructo con el fin de poder obtener confiabilidad y validez.

\section{Educación y discapacidad}

Una de las poblaciones más vulnerables en el sistema educativo son los niños discapacitados. De ahí la preocupación de todos los gobiernos por proporcionarles instrucción. En Estados Unidos de Norteamérica se establece el derecho a una educación pública prescindiendo de cualquier tipo de discapacidad y cualquier problema que obstaculice la capacidad del niño de aprender. Los psicólogos escolares y los docentes de educación especial fueron los primeros profesionales que ayudaron a poner en práctica esta ley. Además, relacionaron lo que se denomina coeficiente emocional con el desempeño académico y el éxito escolar, y elaboraron técnicas y programas para los niños con necesidades especiales (Shapiro, 1997).

En México, el Programa Nacional de Fortalecimiento de la Educación Especial y de la Integración Educativa de la Secretaría de Educación Pública (SEP, 2010), tiene como objetivo promocionar la equidad en el acceso, estabilidad y el logro educativo para los niños(as) y los jóvenes con necesidades especiales. Para lograrlo, proporcionará preferencia a quienes exhiben aptitudes sobresalientes o discapacidad; además, fortificará la atención de estos alumnos en el sistema educativo nacional. Los alumnos que presentan necesidades educativas especiales son 20 786, de éstos, 13470 presentan discapacidad auditiva.

El compromiso de la nación mexicana es asegurar la protección especial para los niños más desaventajados, entre ellos los que tienen discapacidades. Es un imperativo moral y ético que cada programa, producto y país tome en cuenta a los bebés y niños con discapacidades, 
incluyendo a sus familias, como parte de la atención y desarrollo. Una de las discapacidades más frecuentes en nuestro entorno es la discapacidad auditiva. Según datos del estado de Sonora, del registro de los menores con discapacidad, en 1996 había 5865 con discapacidad auditiva; del total, 917 poseían sordera y 4948 debilidad auditiva (Romano \& Pérez, 2007 citado por Castell, Betancourt, Samayoa \& Caballero, 2008). No obstante, existe poca investigación (si no es que ninguna) con esta población y menos aún en lo referente a las temáticas artísticas de inteligencias múltiples, capacidad de liderazgo, comportamientos éticos, inteligencia emocional y salud de estos niños.

\section{Problemática de los niños sordos}

Existe una ley sobre la cultura del sordo a nivel federal; ésta define quién es una persona sorda y qué es una comunidad de sordos. A continuación se transcribe un párrafo de la mencionada ley.

Comunidad de sordos se refiere a toda aquella persona que forma parte de un grupo social que como característica fundamental no posee el oído suficiente para sostener una comunicación y socialización natural y fluida en lengua oral alguna, y que conforme al artículo $2^{\circ}$ de la Constitución Política de los Estados Unidos Mexicanos tiene una cultura propia y posee una lengua de señas que dan sustento al carácter pluricultural de nuestra Nación y que, en tal sentido, son equiparables a los pueblos y comunidades indígenas (Ley Federal de la Cultura del Sordo, 2008)

Las áreas donde se pueden presentar problemas y que, por tanto, requieren atención especial en los niños sordos son el desarrollo comunicativo, lingüístico y social. En este último caso, las investigaciones realizadas parecen indicar que los niños(as) sordos tienen un conocimiento social limitado y, a menudo, presentan problemas en sus habilidades sociales, sobre todo en aquellas que están relacionadas con los procesos atribucionales y las que tiene que ver con la comprensión y expresión de emociones (Gregory, 1976; Liben \& Drury, 1977; Meadow,
1980). Estos datos son fácilmente explicables si se considera la implicación sobre el desarrollo de la competencia social y la comunicación.

La ausencia de comunicación oral implica una menor habilidad para la autorregulación y ciertas limitaciones para extraer el significado de las experiencias cotidianas, lo que a su vez conlleva una menor comprensión de la dinámica social. Todo ello se puede traducir en problemas de autoestima y de conducta, así como de una escasa comprensión de las causas como de los significados de muchos acontecimientos (Ochaita \& Espinosa, 2004).

Los niños(as) sordos tomados en su conjunto y comparados con los que sí oyen, tienen más riesgo de presentar problemas de conducta, de personalidad y de tener complicaciones para alcanzar una buena competencia social. Es decir, exteriorizan más dificultades para controlar sus impulsos, desarrollar un concepto adecuado y autoestima, reconocer y expresar emociones o sentimientos, expresar empatía, evaluar afectos o emociones que resultan de ciertos actos o establecer procesos de atribución de causa entre ciertos acontecimientos (Greenberg, 2003; Meadow, 1980). En un estudio de Castell y colaboradores (2008), la mayoría de las 223 madres de niños sordos consideró que su hijo presentaba buenos modales en general, y que se mostraba empático con frecuencia. Sin embargo, otras de las madres señalaron que su hijo contestaba mal e interrumpía y que sólo en ocasiones era cortés, paciente y esperaba su turno. Estos resultados concuerdan con las investigaciones subsecuentes que dieron la pauta para investigar si se fomentaban o no en la comunidad sorda el desarrollo de comportamientos éticos con base en el arte.

Los niños(as) sordos expresan restringidamente conceptos emocionales, tienen dificultad al expresar los sentimientos y emociones así como relacionarlos verbalmente con sus antecedentes y sus consecuencias. Las características más frecuentementes adjudicadas a los niños sordos son mayor impulsividad, egocen- 
trismo, pobre auto concepto e inmadurez social (Domínguez \& Alonso, 2004).

En el proyecto de Mark Greenberg (2003), en la Universidad de Washington, compararon alumnos regulares con sordos. Los resultados mostraron el aumento de las habilidades cognitivo sociales, como la emoción, el reconocimiento, la comprensión, el dominio de sí mismo, la planificación para solucionar tareas cognitivas, y más reflexión antes de actuar, más eficacia en la resolución de conflictos de los alumnos que participaron comparados con los controles, además, de un clima más positivo en el aula (Goleman, 2005).

Los resultados vertidos en las dos últimas investigaciones son valiosas; primero, porque permite comparar las características de niños sordos de otros países con niños de nuestro estado y, segundo, porque revelan que pese a dichas características, los niños sordos lograrán ajustarse con mayor razón a una intervención no verbal más propia para los niños a través de técnicas psicológicas y artísticas lúdicas.

\section{Aprendizaje y niños sordos}

Shapiro (2003), asegura que el método para que los niños se desarrollen felices, saludables y productivos implica cambiar la forma cómo se desenvuelve el cerebro. De acuerdo con Goleman (2005), las lecciones emocionales (incluso los hábitos más profundamente arraigados, aprendidos en la infancia) pueden ser remodeladas, ya que el aprendizaje emocional dura toda la vida. Por su parte, Béliveau (2002), asegura que es fácil que un niño con alguna discapacidad se sienta responsable de situaciones como la ausencia o enfermedad de uno de los padres, el divorcio, la separación o la falta de recursos económicos. Este tipo de temores suelen traducirse en insomnio o retrocesos como mojar la cama o comportarse como bebé. Variables que pueden modificar considerablemente la situación de los niños discapacitados, sean sordos o no. Estos niños tienen necesi- dades educativas especiales, requieren de recursos materiales y, principalmente, humanos para ser atendidos. La respuesta a tal situación es la educación y la reeducación pertinente en su caso.

Una parte decisiva para la atención de los niños sordos es la educación artística. Lomnitz (2003), asegura que el teatro es una parte fundamental de la cultura del sordo. Por su parte, Ruiz (2009), Hernández (2009) y Rovati (2007), afirman que los niños sordos también pueden disfrutar de la música y beneficiarse positivamente al desarrollar un sentido rítmico. Al respecto, Hernández (2009), en su desempeño como maestra de sensibilización corporal en la Escuela de Artes, descubrió que a través de la danza y las artes, los niños(as) ciegos, con síndrome de Down, con parálisis cerebral y sordos mejoran su capacidad de comunicarse con los demás, de trabajar en equipo y su confianza en sí mismos.

Una de las poblaciones ideales para medir los efectos de la educación artística en el desarrollo de repertorios académicos, creatividad, capacidad de liderazgo, inteligencias múltiples, inteligencia emocional, comportamientos éticos y la salud, son los niños de una comunidad sorda. No obstante, primero resulta necesario elaborar y validar una escala. En este sentido, el objetivo del presente estudio fue presentar el proceso de diseño y validación del instrumento Escala para la Evaluación de la Educación Artística (EEEA).

\section{Método}

\section{Participantes}

Participaron en el estudio 50 padres y 50 maestros de niños(as) sordos que cursaban educación básica en una escuela pública. Los maestros(as) de los niños(as) contaban con una edad mínima de 22 años y una máxima de 55; la media fue de 37.56 . El $90.5 \%$ de ellos recibió educación artística durante su infancia y $9.5 \%$ no la recibió. El 13\% tomó cursos de educación 
artística y $23.8 \%$ no tomó ningún curso sobre la temática. En relación con el nivel académico de los maestros entrevistados, 41 contaban con licenciatura, 8 con maestría, y uno había cursado preparatoria. En licenciatura había normalistas en Educación y en Educación especial, en Psicología, en Danza, en Artes, en Educación física y en Matemáticas.

Los padres de los niños (as) contaban con una edad mínima de 22 años y una edad máxima de 51 años; la media de edad fue de 34.88. El $86.9 \%$ de los padres recibió educación artística durante la infancia y $9.5 \%$ no la recibió. El $16.7 \%$ tomó un curso de educación artística y $44.0 \%$ no tomó ningún curso sobre la temática. En relación con el nivel académico de los padres de los niños(as) entrevistados, 25\% contaba con licenciatura, 31\% preparatoria, 38\% secundaria y sólo $6 \%$ estudió la primaria. Las licenciaturas cursadas eran Biología, Medicina, Química, Enfermería, Trabajo social, Ingeniería y Contaduría; el $8 \%$ cuenta con carreras técnicas: bombero, comercio, estilista, asistente educativa, costurera, asistente médico, jornalero, secretaria. El $60 \%$ se dedicaba al hogar, 33\% trabajaba y $7 \%$ era estudiante.

\section{Instrumento}

Para la elaboración del EEEA se consideró la opinión de expertos y se realizó una revisión de bibliografía sobre la temática, así como de algunos instrumentos como la Escala de Ambientes Escolares Revisada (Environment Rating Scale Revised, ITERS-R), la Escala del Ambiente de Edad Escolar (School Aged Environment Rating Scale, SACERS), y la Escala de Evaluación de Contextos Educativos Infantiles (Early Childhood Environment Rating Scale Revised, ECERS-R) de Harms y Clifford, (1980). Cada subescala contenía reactivos que median el ambiente físico, el cuidado básico del niño, el currículo, las interacciones, estructura del programa, y las necesidades de los padres y del personal. Cada una de ellas diseñada para un segmento diferente del desarrollo del niño(a).

La escala original ECERS de Harms y Clifford fue publicada en 1980 y contenía sólo 7 subescalas y 37 reactivos. La ECERS-R (Harms, et al. 1998), también contenía 7 subescalas y aumentó sus reactivos a 43 en total. Un cambio adicional en la transición de la escala ECERS a la ECERS-R fue que esta última puso un gran énfasis en algunos temas importantes y emergentes en las estancias infantiles, tales como los asuntos familiares, diversidad cultural, y la inclusión de niños con discapacidades, además del uso de un sistema de indicadores más estricto en apoyo al índice de audiencia del uso de la escala ECERS-R.

Al reorganizarse las subescalas en la versión $\mathrm{R}$ (revisada), el alfa de Cronbach reportada para esta adaptación fue mucho más alta que la obtenida por la escala ECERS original (.86). Las alfas reportadas de la escala ECERS-R fueron desde .71 a .88, mientras que en el total de la escala la consistencia interna fue de .92 (Harms, et al., op.cit). A nivel de reactivo, el porcentaje de acuerdo exacto entre calificadores fue de $48 \%$.

Los autores de la escala ECERS-R ofrecen de tres a cinco días de trabajo intensivo para ayudar a los aplicadores a familiarizarse con su uso. Adicionalmente, utilizan videos y están disponibles materiales de entrenamiento y guías con información profunda acerca de cada reactivo e indicador. Los creadores de la escala recomiendan una certificación para su uso, y consideran importante que los usuarios tengan una comprensión completa del sistema de puntuación y del significado de cada indicador para poder obtener confiabilidad de dicho sistema; asimismo, es requerido un entrenamiento extensivo, así como un seguimiento para poder obtener medidas confiables entre múltiples calificadores (Cassidy, Hestenes, Hegde, Hestenes, \& Mims, 2005). 
María José Lera y Ruth Oliver en el 2002, realizaron la traducción de la escala ECERS-R (Early Childhood Environment Rating Scale- Revised Edition), publicada en 1998 por Teacher College Press. De esa versión en español se retomaron tres subescalas para organizar la EEEA, que finalmente quedó dividida en dos secciones: I) Sección institución y II) Sección alumno(a). La sección institución se conformó de las subescalas: 1) características de la institución, 2) características del aula, 3) actividades artísticas, y 4) estructura del programa. A las cuatro se les hicieron ligeras adaptaciones; aumentando o eliminado algunos reactivos. Para la sección alumno(a) se construyeron cuatro subescalas: 1) características del alumno, con sus respectivas dimensiones (académicas, creatividad, artísticas, inteligencias múltiples, liderazgo, comportamientos éticos, inteligencia emocional y salud), 2) características de los padres, 3) características del maestro(a) y 4) interacción con un total de 135 ítems.

Las dos secciones de la escala se concretan en varios indicadores observables y evaluables; a su vez, cada indicador está definido por cuatro criterios de evaluación. El primer criterio describe una calidad "inadecuada"; si esto se observa se puntúa como 1; el segundo criterio describe unas condiciones "mínimas" y se puntúa como 3; el tercer criterio describe unas condiciones de calidad "buena" y le corresponde la puntuación de 5; por último, el cuarto criterio describe unas condiciones "excelentes" y se puntúa como 7. Las puntuaciones intermedias (2, 4 y 6$)$ se otorgan cuando el criterio superior no se cumple en su totalidad. Resumidamente, la escala está compuesta de 135 indicadores, cada uno de ellos se puntúa de 1 a 7 . Estas puntuaciones sirven para obtener la media correspondiente a cada subescala $y$, consecuentemente, se obtiene una valoración de su calidad (inadecuada, mínima, buena o excelente); finalmente, la puntuación global será el indicador de la calidad. La utilización de esta escala con fines de investigación requirió la formación de observadores para garantizar la fiabilidad y validez de los resultados encontrados. Esta evaluación se complementa con la observación directa de las actividades que tienen lugar en el aula.

\section{Procedimiento}

La escala se aplicó a maestros y padres de niños(as) sordos. Los instrumentos fueron administrados por psicólogos y estudiantes avanzados de Psicología. La aplicación se realizó en la escuela de los niños o en sus hogares y duró alrededor de 20 minutos. A todos se les explicó los objetivos de la investigación y que la información era totalmente confidencial. Además, de que podían dejar de contestar el cuestionario en cualquier momento si así lo deseaban.

\section{Análisis de datos}

Primeramente, se realizó una revisión de cada una de las respuestas de los maestros(as) y de los padres de los niños(as) sordos, además, se hicieron categorías para la descripción cualitativa de los resultados y la depuración y mejora del instrumento.

Adicionalmente, estadísticas univariadas fueron calculadas, medias y desviaciones estándar para variables continuas y frecuencias para las variables categóricas, así como alfas para medir la confiabilidad de las escalas.

\section{Resultados}

El proceso de prueba de la EEEA incorporó 135 reactivos. Cinco reactivos de la escala que no resultaron pertinentes fueron eliminados, por lo tanto, éstos se redujeron de 140 a 135. Las tablas 1 y 2 muestran las alfas obtenidas en cada una de las subescalas que conforman las dos secciones: institución y alumno(a) del EEEA, tanto las vertidas por los datos de los maestros, así como las proporcionadas por los datos de los padres. La tabla 1 muestra las al- 
fas de la sección institución con las subescalas: características de la institución, aula, actividades artísticas y estructura del programa; tanto las alfas vertidas con los datos de los maestros, así como las proporcionadas por los datos de los padres.

Tabla 1.

Alfas de la sección institución datos de maestros y padres

\begin{tabular}{lccc}
\hline \multicolumn{1}{c}{ Escala } & $\begin{array}{c}\text { Alfa de Cronbach } \\
\text { Maestros }\end{array}$ & $\begin{array}{c}\text { Alfa de Cronbach } \\
\text { Padres }\end{array}$ & No. ítems \\
\hline Características de la Institución & .84 & .85 & 10 \\
Características del Aula & .89 & .89 & 10 \\
Actividades Artísticas & .90 & .89 & 10 \\
Estructura del Programa & .96 & .97 & 10 \\
\hline
\end{tabular}

Los valores de las alfas de las escalas analizadas oscilan de .84, obtenida para institución, de los datos proporcionados por los maestros a .97, derivada del programa, de los datos vertidos por los padres. Los resultados muestran que para ambos fueron los mismos factores: actividades artísticas (10 reactivos) y estructura del programa (10 reactivos), los que obtuvieron los más altos puntajes de consistencia interna.

La tabla 2 muestra las alfas de la sección alumno(a) tales como: académicas, creativas, artísticas, inteligencias múltiples, capacidad de liderazgo, inteligencia emocional, comportamientos éticos y salud.

Tabla 2.

Alfas de las subescalas características del alumno, datos maestros y padres

\begin{tabular}{lccc}
\hline \multicolumn{1}{c}{\begin{tabular}{c} 
Escala Características \\
\multicolumn{1}{c}{ Alumno }
\end{tabular}} & $\begin{array}{c}\text { Alfa de Cronbach } \\
\text { Maestros }\end{array}$ & Alfa de Cronbach & No. Ítem \\
\hline Efectos del Programa & .94 & .92 & 7 \\
Creatividad & .93 & .93 & 16 \\
Artísticas & .90 & .86 & 5 \\
Inteligencias múltiples & .95 & .91 & 13 \\
Capacidad de liderazgo & .90 & .87 & 9 \\
Comportamientos éticos & .87 & .75 & 5 \\
Inteligencia emocional & .85 & .78 & 3 \\
Salud & .81 & .81 & 7 \\
\hline
\end{tabular}

Los valores de las alfas de las escalas analizadas parecen revelar consistencia interna dado que las alfas oscilan de .75, obtenida por comportamientos éticos, de los datos proporcionados por los padres a .95, emanada por inteligencias múltiples de los datos vertidos por los maestros. Los resultados muestran que para ambos maestros y padres resultaron los mismos factores: efectos del programa (10 reactivos) e inteligencias múltiples (13 reactivos), 
los que obtuvieron los más altos puntajes de consistencia interna. La tabla 3 muestra las alfas de las subescalas: evaluación a padres, evaluación a maestros e interacción de la sección alumno(a).
Los valores de las alfas de las escalas analizadas revelan consistencia interna, dado que los valores oscilan de .87 , obtenida por evaluación a maestros a .95, obtenida por evaluación a padres.

Tabla 3.

Alfas de las escalas evaluación a padres, evaluación a maestros e interacción, datos maestros y padres

\begin{tabular}{lccc}
\hline \multicolumn{1}{c}{ Escala } & $\begin{array}{c}\text { Alfa de Cronbach } \\
\text { Maestros }\end{array}$ & $\begin{array}{c}\text { Alfa de Cronbach } \\
\text { Padres }\end{array}$ & No. Ítems \\
\hline Evaluación a padres & .95 & .95 & 10 \\
Evaluación a maestros & .91 & .87 & 10 \\
Interacción & .89 & .89 & 10 \\
\hline
\end{tabular}

\section{Discusión}

La versión completa de la EEEA fue aplicada a una muestra de maestros(as) de educación básica que laboran en escuelas públicas y de padres de niños sordos de los diferentes puntos del estado. Los resultados mostraron que las escalas presentaban consistencia interna. Por lo tanto, se pueden considerar confiables para medir la conducta y poder hacer predicciones. El efecto de la educación artística en la conducta de los niños necesita ser evaluada, por lo tanto, este instrumento va a resultar útil en ese tipo de mediciones. El mejoramiento de la educación requiere de estas evaluaciones, de ahí que se requieran instrumentos confiables para realizarlas. Consecuentemente, se considera que el aporte fundamental es haber contribuido al desarrollo de una escala cuyos ítems reflejan fielmente los indicadores esenciales de la educación artística.

En este sentido, se busca generar instrumentos valiosos y también proporcionar una base empírica sólida para el estudio de la educación artística. Es de llamar la atención la inexistencia de trabajos dedicados a evaluar la educación artística, por lo cual se requiere implementar investigaciones sobre la valoración de esta materia, con las cuales se pudiera conocer:

- El estado del arte de la educación artística en el estado y en la comunidad.

- La formación y el grado de especialización en el área con los cuales cuentan los maestros.

- La calidad de la educación artística (institución, programa, aula, actividades artísticas).

- Los efectos de la educación artística en las características del niño (académicas, creativas, artísticas, inteligencias múltiples, capacidad de liderazgo, inteligencia emocional, comportamientos éticos y salud).

- Las características de los maestros y de los padres en términos de la calidad de estimulación y modelamiento proporcionados. Analizar el rendimiento académico, la creatividad, el gusto por el arte, el dominio de algún tipo de arte, el fomento de la práctica de actividades artísticas extracurriculares, la estimulación de las inteligencias múltiples, el modelamiento de la capacidad de lide- 
razgo, la inteligencia emocional, los comportamientos éticos, la incorporación de los métodos que inciten inteligencias múltiples en el niño(a) y la interacción adecuada de maestros y padres con los niños(as).

Las evaluaciones permitirán recuperar las experiencias, aportaciones y observaciones realizadas por los investigadores que participan en el trabajo, además, contribuirán a la construcción de las propuestas de intervención educativa.

La concepción sobre la educación implica no sólo el diagnosticar a tiempo posibles problemas académicos, sino darles una orientación y solución adecuada. Por lo tanto, el diseño de instrumentos que analicen la inclusión de las temáticas de arte en la educación y la implementación de esta materia en el currículo oficial de los niños de educación básica es un paso importante para el impulso de su desarrollo intelectual.
Alianza por la Calidad de la Educación ACE (2008). Recuperado de www.sep.gob.mx/es/sep1/alianzaporlacalidaddelaeducacion

Béliveau, M. (2002). J'ai mal a'l'école. Troubles affectifs et difficultés scolaires. Canadá: Hópital Sainte-Justine Editions.

Cassidy, D., Hestenes, L., Hedge, A., Hestenes, S., \& Mims, S. (2005) Measurement of quality in preschool child care classrooms: The early childhood environment rating scale. Revised and it's psychometric properties. Early Childhood Research Quarterly, 20 (3), 345-360.

Castell, I., Betancourt, R., Samayoa, L., \& Caballero, O. (2008). Inteligencia emocional y personalidad en niños sordos. León Guanajuato: Memorias del XV Congreso Asociación Mexicana de Psicología Social.AMEPSO.

Delors J. (2009). La educación encierra un tesoro. Compendio. Informe a la UNESCO de la Comisión Internacional sobre la educación para el siglo XXI. París, Francia: Santillana Ediciones UNESCO.

Destrempes-Marquez, D.y Lafleur, L. (2003).Lestroublesd'apprentissage: comprendere e intervenir. Québec, Canadá: HópitalSainte-Justine.

Domínguez, A \& Alonso, P. (2004). La educación de los alumnos sordos hoy. Perspectivas y respuestas educativas. Archidona, Málaga, España: Ediciones Aljibe.

Frías, M. \& Corral V. (2001) Evaluación de Programas de Intervención. Hermosillo Sonora, México: Editorial UNISON.

Gardner, H. (2006) Changing Minds. The art and science of changing our own and other people's minds. Boston, MA: Harvard Business School Press.

Gardner, H. (2010). Mentes creativas: Una anatomía de la Creatividad. Barcelona España: Ediciones Paidós Ibérica.

Gento, S. (2002). Instituciones educativas para la calidad total: configuración de un modelo organizativo. Madrid: Editorial La Muralla.

Goleman, D. (2005). Emociones Destructivas. Barcelona, España: Editorial Vergara.

Greenberg, M. (2003). PHATS (Promoting Alternative Thinking Strategies). Recuperado de http://prevention.psu.edu/documents/ Project_Flyer_000.pdf

Gregory, S. (1976). The deaf child and his family. London: Allen and Unwin.

Harms, T. \& Clifford, R.M. (1980). Early Childhood Environmental Rating Scale. New York, NY: Teachers College Press.

Hernández, C. (2009). Sensibilización Corporal y Experiencias Dancísticas. Escuela de Artes. Puebla, México: BUAP y Colectivo Poético Ricardo Hernández Huerta.

Jacinto, C. \& Gallant, M. (1998). La Evaluación de programas de capacitación de jóvenes desfavorecidos: una ilustración con programas para jóvenes desempleados en los países del Cono Sur. Documento de trabajo. Serie estrategias de Educación y de formación para los grupos desfavorecidos. París: UNESCO.

Ley Federal de la Cultura del Sordo (2008). Recuperado de www.cddhcu. gob.mx

Liben, L. \& Drury, D. (1977). Short -term memory in deaf and hearing children in relation to stimulus characteristics. Journal of experimental child psychology, 24, 60-73. Recuperado de http://www.basica.sep. gob.mx

Lomnitz, A. (2003). Dibujar en el aire. En R. Castro (Ed.), Las otras lecturas. México: Editorial Paidós.

Meadow, K. (1980).Deafness and child development. Londres: Edward Arnold.

OECD (2002). (8 de Julio de 2008) Development assistance committee, glossary of key terms in evaluation and RBM. Recuperado en http:// www.sida.se/sida.jsp?d=118Ga=3148\&languaje

Ochaita, E. \& Espinosa, M. (2004). Hacía una teoría de las necesidades infantiles y adolescentes. Necesidades y derechos en el marco de la convención de Naciones Unidas sobre derechos del niño. UNICEF. Aravaca, Madrid, España: Editorial McGraw Hill.

Passenger, T., Stuart, M., \& Terrel, C. (2003). Phonological processing and early literacy. Journal of Research in Reading, 23 (1), 55-66. 
Plan Nacional de Desarrollo, PND (2006). (8 de enero del 2008) Recuperado en http://www.lib.utexas.edu/benson/lagovdocs/ mexico/federal/presidente/pnd/PNDmex01-06.pdf

Programa para la evaluación internacional de los alumnos PISA (2003). (28 de mayo del 2009) Informe PISA 2003. Aprender para el mundo de mañana. Recuperado de http://www.oecd.org/ dataoecd/59/1/39732493.pdf

Programa para la evaluación internacional de los alumnos PISA (2006). Marco de la Evaluación. Conocimientos y Habilidades en Ciencias, Matemáticas y Lectura. Santillana Educación S. L. Obra publicada por acuerdo con la OECD.

Programa Sectorial de Educación PROSEDU (2007-2012). (20 de enero del 2010). Recuperado de http://www.ithua.edu.mx/documentos/ prosedu_2007_2012.pdf

Rossi, P. \& Freeman, H. (1985). Evaluation: a systematic approach. Thousand Oaks, CA: Sage publications.

Rovati L. (2007). Musicoterapia para niños sordos. Recuperado de http:// medranoantonia64.blogspot.com/2008/10/musicoterapia-para-niossordos-lola.html

Ruiz, Y. (2009). La Musicoterapia en la Educación Especial. Recuperado de www.educacionmusical.com

Secretaría de Educación Pública (SEP). (2010). Recuperado de http:// www.sep.gob.mx

Shapiro, L. (1997). La inteligencia emocional de los niños. Barcelona, España: Editorial Vergara.

Shapiro, L. (2003). El Lenguaje Secreto de los niños. Barcelona, España: Editorial Urano. 